\title{
THE
}

\section{Drag Effect and Topological Complexes in Strongly Interacting Two-Component Lattice Superfluids}

\author{
V. M. Kaurov
}

A. B. Kuklov

A. E. Meyerovich

University of Rhode Island, sfo101@uri.edu

Follow this and additional works at: https://digitalcommons.uri.edu/phys_facpubs

Terms of Use

All rights reserved under copyright.

\section{Citation/Publisher Attribution}

Kaurov, V. B., Kuklov, A. B., \& Meyerovich, A. E. (2005). Drag Effect and Topological Complexes in Strongly Interacting Two-Component Lattice Superfluids. Phys. Rev. Lett., 95, 090403. doi: 10.1103/

PhysRevLett.95.090403

Available at: http://dx.doi.org/10.1103/PhysRevLett.95.090403

This Article is brought to you for free and open access by the Physics at DigitalCommons@URI. It has been accepted for inclusion in Physics Faculty Publications by an authorized administrator of DigitalCommons@URI. For more information, please contact digitalcommons-group@uri.edu. 


\title{
Drag Effect and Topological Complexes in Strongly Interacting Two-Component Lattice Superfluids
}

\author{
V. M. Kaurov, ${ }^{1}$ A. B. Kuklov, ${ }^{1}$ and A. E. Meyerovich ${ }^{2}$ \\ ${ }^{1}$ Department of Physics, CUNY - Staten Island, New York, New York 10314, USA \\ ${ }^{2}$ Department of Physics, University of Rhode Island, Kingston, Rhode Island 02881, USA
}

(Received 5 May 2005; published 26 August 2005)

\begin{abstract}
The mutual drag in strongly interacting two-component superfluids in optical lattices is discussed. Two competing drag mechanisms are the vacancy-assisted motion and proximity to a quasimolecular state. In a case of strong drag, the lowest energy topological excitation (vortex or persistent current) can consist of several circulation quanta. In the SQUID-type geometry, the circulation can become fractional. We present both the mean field and Monte Carlo results. The drag effects in optical lattices are drastically different from the Galilean-invariant Andreev-Bashkin effect in liquid helium.
\end{abstract}

DOI: 10.1103/PhysRevLett.95.090403

The observation [1] of the quantum phase transition [2] between superfluid (SF) and Bose Mott insulator in optical lattice (OL) has stimulated strong interest toward new quantum phases in multicomponent systems [3-8]. Two recent examples include topological excitations - vortices and persistent currents with nonstandard winding properties in two-component superfluids (2SF) [5,6].

A crucial but largely unaddressed effect is the impact of strong interaction on the properties of superfluid phases where each component $\psi_{a}$ has its finite expectation value $\left\langle\psi_{a}\right\rangle$. A direct manifestation of the strong interaction is the intercomponent drag similar to the Andreev-Bashkin effect [9] in 2SF helium mixtures. The drag between nonconvertible species at zero temperature is represented by the cross terms in the expansion of the ground state energy in terms of small gradients of the superfluid phases $\nabla \varphi_{a}, a=1,2$,

$$
\delta E=\int d \mathbf{x}\left[\frac{1}{2} \sum_{a, b} \rho_{a b} \nabla \varphi_{a} \nabla \varphi_{b}\right],
$$

with $\rho_{a b}$ standing for the superfluid stiffnesses. The cross term $\rho_{12}$, responsible for the drag, is due to interaction effects. Depending on its sign, this term describes either a mutual unidirectional flow or a counterflow of the components. The drag effect is qualitatively different from trivial dynamics of mixing driven by kinetic pressure gradients $\sim \mathbf{j}_{a}^{2} \nabla N_{a}$, with $\mathbf{j}_{a}$ and $N_{a}$ being currents and densities, respectively. While the drag is linear in $\mathbf{j}_{a}$, the mixing dynamics is quadratic on $\mathbf{j}_{a}$. This can help distinguishing the drag from the mixing in nonuniform OL. In general, the pressure/density gradients, which are related to the inhomogeneity of the trap, affect the drag leading, eventually, to the tensor rather than scalar nature of the effect in Eq. (1). However, these effects are negligible as far as the density does not experience noticeable changes on the period of OL.

The Galilean invariance imposes two constraints on $\rho_{a b}$. These constraints are responsible for the Andreev-Bashkin effect in superfluid mixtures of liquid helium isotopes in
PACS numbers: 03.75.Kk, 03.75.Lm, 03.75.Mn, 67.60.Hr which $\rho_{12}$ is uniquely related to the ratio of bare $m_{1}$ and effective $m_{1}^{*}$ atomic masses. In a frame moving with velocity $\mathbf{V}$ the phase of each component changes as $\varphi_{i} \rightarrow$ $\varphi_{i}-\left(m_{i} / \hbar\right) \mathbf{V} \cdot \mathbf{r}$, where $m_{i}$ are the bare masses. The energy density (1) transforms as $\delta E \rightarrow \delta E-\mathbf{P V}$, where $\mathbf{P} / \hbar=N_{1} \nabla \varphi_{1}+N_{2} \nabla \varphi_{2}$ is the momentum density. This yields

$$
\rho_{11} m_{1}+\rho_{12} m_{2}=N_{1}, \quad \rho_{12} m_{1}+\rho_{22} m_{2}=N_{2} .
$$

Introducing effective masses $m_{1}^{*}, m_{2}^{*}$ as $\rho_{11}=N_{1} / m_{1}^{*}$, $\rho_{22}=N_{2} / m_{2}^{*}$, we reproduce the result [9], $\rho_{12}=$ $\left(N_{1} / m_{2}\right)\left(1-m_{1} / m_{1}^{*}\right)$ as well as the relation $N_{1}\left(m_{1}-\right.$ $\left.m_{1}^{*}\right) m_{1} / m_{1}^{*}=N_{2}\left(m_{2}-m_{2}^{*}\right) m_{2} / m_{2}^{*}$. In other words, the conservation of the total momentum requires that the difference between bare and effective masses is compensated by the flux of the other component. Note that $\rho_{12}>0$ since $m_{1,2}^{*}>m_{1,2}$.

In the case of strong mass renormalization, $\left(m_{1}^{*} / m_{1}\right)-$ $1 \geq 1$, quite spectacular effects should be expected [10] from vortices. Specifically, the lowest energy singlecirculation vortex of the majority component $\left(\rho_{22} \gg\right.$ $\left.\rho_{11}\right)$ should carry several circulation quanta $q=1,2, \ldots$ of the minority component. The equilibrium value of $q$ is obtained by minimizing the factor $m_{2} q^{2}+2\left(m_{1}^{*}-m_{1}\right) q$ in the energy of the vortex complex (or persistent current). These $q+1$ vortex complexes exhibit transformations with respect to the value of $q$ depending on external conditions that determine $m_{1}^{*}$.

In this Letter, we address the drag effect in a lattice $2 \mathrm{SF}$ in strongly interacting limit [when no expansion in the gas parameter can be employed [11] ], and show that the lattice plays a central role in violating the relation [9] between $\rho_{12}$ and $m_{1} / m_{1}^{*}$ [and the constraints (2)]. We also argue that the value of $q$ is affected by proximity of the $2 \mathrm{SF}$ to the quasimolecular phase.

In OL, in contrast to the Galilean-invariant system, the lattice provides a preferred reference frame, so that the (hydrodynamic) properties of the two-component mixture 
are determined not by the relative velocity of components but by their individual velocities with respect to the lattice. Furthermore, the effective mass in OL is formed largely by the width and depth of laser-generated potential wells rather than by a trailing cloud of the second component. Another crucial difference is that in OL the number of vacancies is a conserved quantity. Below we perform the mean field and Monte Carlo analysis of the mutual drag in 2SF in three different physical situations: a soft-core system close to molecular condensation, a hard-core system with finite intercomponent exchanges, and a hard-core system with vacancy-assisted motion without the intercomponent exchanges.

Drag due to proximity to the quasimolecular state.Here we discuss a generic mechanism leading to the $q+1$ topological complexes in the 2SF. Strong drag effect occurs if a two-component boson system is close to a transition into the quasimolecular state in which the only broken symmetry has the order parameter $\Phi_{q} \sim$ $\exp \left(i \varphi^{(q)}\right) \sim\left\langle\psi_{1} \psi_{2}^{q}\right\rangle \neq 0$ (or $\left\langle\psi_{1} \psi_{2}^{\dagger q}\right\rangle \neq 0$ ). In a pure molecular state with undefined individual phases $\varphi_{1,2}$ (that is, $\left\langle\psi_{1,2}\right\rangle=0$ ), the phase-gradient energy is given by the molecular superfluid phase $\varphi^{(q)}$ as $\delta E=$ $\int d \mathbf{x} \rho_{q}\left(\nabla \varphi^{(q)}\right)^{2} / 2$, with $\rho_{q}$ being the molecular superfluid stiffness. The molecular order parameter persists in the 2SF phase so that the additional broken $U(1)$ symmetry emerges continuously [6]. The two phases $\varphi_{1,2}$ become well defined in the 2SF state with the molecular phase being locked as

$$
\varphi^{(q)}=\varphi_{1}+q \varphi_{2}
$$

This locking can be understood as a consequence of virtual processes of transformation of a $(q+1)$ molecule into $q B$

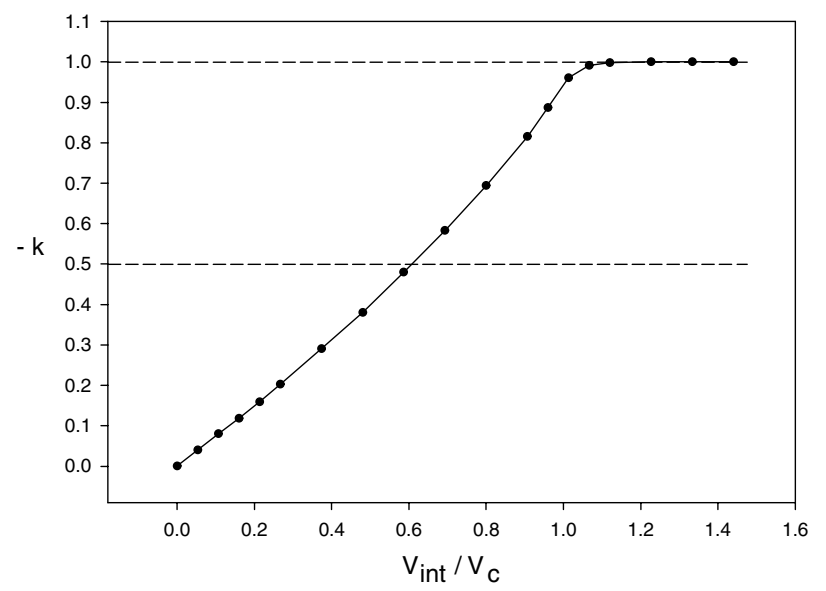

FIG. 1. The drag coefficient $k$ for the $J$-current analog of the Hamiltonian (6) as a function of the relative interaction, with the value $V_{\text {int }} / V_{c}=1$ corresponding to the $2 \mathrm{SF}-\mathrm{SCF}$ phase transition. The horizontal dashed lines indicate a domain where $1+q$ vortex complex with $q=1$ has lower energy than any singlecirculation vortex. Error bars are much smaller then symbol sizes. Solid line is the eye guide. atoms and one $A$ atom. The corresponding contribution to the energy functional is $\Delta E \sim \int d \mathbf{x} \Phi_{q} \psi_{1}^{*} \psi_{2}^{* q}+$ H.c.. This term [cf. the diatomic molecules with $q=1$ [12] ] ensures the relation (3) in the long-wave limit. Then the energy (1) becomes

$$
\begin{aligned}
\delta E= & \int d \mathbf{x}\left[\frac{\rho_{q}}{2}\left(\nabla\left(\varphi_{1}+q \varphi_{2}\right)\right)^{2}+\frac{\rho_{1}^{\prime}}{2}\left(\nabla \varphi_{1}\right)^{2}\right. \\
& \left.+\frac{\rho_{2}^{\prime}}{2}\left(\nabla \varphi_{2}\right)^{2}+\rho_{12}^{\prime} \nabla \varphi_{1} \nabla \varphi_{2}\right],
\end{aligned}
$$

with $\rho_{i j}^{\prime}$ continuously changing from zero in the molecular phase to some finite values in the $2 \mathrm{SF}$ phase. It is important that the molecular stiffness $\rho_{q}$ is not a critical property of the system-it does not change while crossing the phase boundary. Thus, at least close to the phase boundary, minimization of the vortex energy gives $\varphi_{1}=-q \varphi_{2}$, that is, the $q+1$ vortex. In reality, the relations $\left|\rho_{a b}^{\prime}\right| \ll$ $\left|\rho_{12}\right| \approx \rho_{1,2}$ can hold quite far from the phase boundary. This implies that the $q+1$ topological excitation exists deep in the 2SF phase. We demonstrate this numerically for $q=1$ (see Fig. 1 below).

It is convenient to introduce the drag coefficient $k$ as a ratio $k=\rho_{12} / \rho_{11}$ of the cross stiffness to the smallest diagonal stiffness, $\rho_{11} \leq \rho_{22}$. Then, as the minimization of the energy (1) shows, when $|k|>0.5$, a vortex of the dominant component can lower its energy if it carries the circulation of the other component $q= \pm 1$. In symmetric case $\left(\rho_{11}=\rho_{22}\right)$, the integer $q$ closest to $k$ determines the $q+1$ vortex (or persistent current) as the minimal topological excitation. It is important to note that even small $|k|$ causes attraction between either vortices of equal circulations $(k<0)$ or between vortex and antivortex $(k>0)$ in different components, so that if both exist they will form a complex. Crossing the boundary $|k|>0.5$ has strong impact on mechanisms of vortex creation and stability. For example, stirring the component with the largest stiffness $\left(\rho_{22}\right)$ above the threshold will cause creation of the complex instead of a single vortex of the stirred component. Also, a single vortex of the component 2 becomes unstable with respect to inducing creation of a vortex of the other component.

The Hubbard lattice model with molecular phases,

$$
H=\sum_{\alpha,\langle i j\rangle}\left[-t_{\alpha} a_{\alpha, i}^{\dagger} a_{\alpha, j}+\text { H.c. }\right]+\sum_{\alpha, \alpha^{\prime}, i}\left[U_{\alpha, \alpha^{\prime}} n_{\alpha i} n_{\alpha^{\prime}, i}\right],
$$

has been extensively studied analytically $[4,8]$ and numerically $[6,7]$. Here $U_{\alpha, \alpha^{\prime}}$ is the interactions matrix, $t_{\alpha}$ describes the nearest-neighbor jumps of component $\alpha$, $a_{\alpha, i}^{\dagger}, a_{\alpha, j}$ are the construction bosonic operators, and $n_{\alpha i}=$ $a_{\alpha, i}^{\dagger} a_{\alpha, i}$ are the on-site occupancies. As discussed in Refs. [6,7], the quasimolecular phase $\left(U_{12}<0\right)$, namely, the paired superfluid, is in many respects isomorphic to the supercounterfluid state $\left(U_{12}>0\right)$ [4]. Both states can undergo second order phase transition into the $2 \mathrm{SF}$ phase 
so that the order parameter $\Phi_{q=1}=\left\langle\psi_{1} \psi_{2}\right\rangle$ (or $\Phi_{q=1}=$ $\left.\left\langle\psi_{1} \psi_{2}^{\dagger}\right\rangle\right)$ remains finite and robust. Obviously, in the 2SF phase, the $q=1$ composite vortices are the lowest topological excitations. As pointed out in Ref. [13], the Hamiltonian (5) also allows molecular phases with an arbitrary integer value of $q$. This issue, though, requires separate analysis.

Hard-core limit $U_{a b} \rightarrow \infty$ of the Hamiltonian (5). - This limit exhibits quite interesting physics of strong quantum fluctuations even far from any phase transition [5]. Obviously, when $N_{A}+N_{B}=1\left(N_{A}, N_{B}\right.$ are the average on-site occupancies of the species $A, B)$, the system in the hard-core limit (HC) is the Mott insulator. Its ground state is degenerate with respect to possible permutations of bosons $A$ and $B$. This degeneracy, which is a consequence of the $\mathrm{HC}$ approximation, is lifted by any infinitely small intercomponent exchanges. Accordingly, the twocomponent HC model should be considered as a limit of the model in which the intercomponent interaction $V_{\text {int }}=$ $U_{12}$ is finite. In contrast to free space, increasing $V_{\text {int }}$ leads to decrease of all superfluid stiffnesses because all transport is suppressed as $\sim t_{1} t_{2} / V_{\text {int }}$. This is clearly at variance with the Galilean constraints (2) which prohibit uniform decrease of all stiffnesses at fixed densities.

The two-component Hamiltonian with soft-core intercomponent repulsion is represented in terms of the $\mathrm{HC}$ construction operators $a_{i}^{\dagger}, a_{i}$ and $b_{i}^{\dagger}, b_{i}$ with Pauli commutation relations for the $A$ and $B$ components

$$
H=\sum_{\langle i j\rangle}\left[-t_{1} a_{i}^{\dagger} a_{j}-t_{2} b_{j}^{\dagger} b_{i}+\text { H.c. }\right]+\sum_{i} V_{\mathrm{int}} a_{i}^{\dagger} a_{i} b_{i}^{\dagger} b_{i}
$$

with summation $\langle i j\rangle$ over the nearest-neighbor sites. At total filling 1, this Hamiltonian has two phases-2SF, where both SF order parameters are defined, and supercounterfluid (SCF), where the only SF order is observed in $\left\langle a_{i} b_{j}^{\dagger}\right\rangle$.

Transition between these two phases is continuous in the universality class $U(1)$ [6] and occurs in the symmetric case $t_{1}=t_{2}=t$ at some value $V_{\text {int }}=V_{c}, V_{c} / t \sim 1$. As discussed above, the drag effect is strong in the 2SF phase even far from the transition. We proved this by performing the Worm algorithm [14] Monte Carlo simulations of the two-color $J$-current model $[6,7,15]$ at zero temperature on a $2 D$ square lattice. This model is a discrete-time grandcanonical analog of the Hamiltonian (6) with the hard-core constraints. The stiffnesses were determined from the statistics of the winding numbers similarly to Refs. $[6,7,16]$. The SCF phase was identified by observing $\rho_{11}=\rho_{22}=$ $-\rho_{12}$. The negative value of $\rho_{12}$ is due to counterflow of the components - each winding of $A$ world line is accompanied by opposite winding of $B$ world line. In Fig. 1, the drag coefficient $k$ is plotted as a function of the relative interaction strength. As can be seen, the domain $1 / 2<$ $|k|<1$ in the $2 \mathrm{SF}$ (between the dashed lines), where the composite $1+q$ vortex with $q=1$ has lower energy than any single vortex, is not restricted to the vicinity of the critical point $V_{c}$ but occupies about half of the phase diagram. Here $\rho_{12}<0$, indicating that both components participate in the counterflow even in the $2 \mathrm{SF}$ state.

Vacancy-assisted drag. - If the total filling is different from 1, the system is always in $2 \mathrm{SF}$ phase at $T=0$. In this case, another mechanism contributes to the drag-the vacancy-assisted transport. Atoms tunnel to the unoccupied sites (vacancies) much faster than the rate of the $A-B$ exchange with large $V_{\text {int }}$. The vacancies stimulate the mass flow in one direction and move in the opposite one. As a result, both components $A$ and $B$ move in one direction, which means that $\rho_{12}>0$. This implies crossover when $\rho_{12}$ changes sign at some point with no drag, $\rho_{12}=0$. Since no symmetry change takes place, this is not a phase transition. The crossover from $k<0$ to $k>0$ takes place as $V_{\text {int }}$ increases at fixed number of vacancies.

The drag coefficient $k$ must increase when the number of vacancies $x_{v}=1-N_{A}-N_{B}$ decreases. This counterintuitive result stems from the nature of vacancies. In one component case, conservation of the number of vacancies $N_{V}$ makes them similar to particles. The HC limit links the flow of vacancies with the opposite flow of atoms. In the two-component case, the situation is similar with one crucial difference - a vacancy is not uniquely associated with a particular sort of atoms. Thus, motion of a single vacancy through a lattice in one direction leads to flows of both components in the opposite direction. This implies strong drag with positive $k$. When $x_{v}$ increases, the system becomes more like a low density and, thus, weakly interacting mixture of two sorts of atoms with a correspondingly small $k$.

To analyze the mutual drag and the possibility of complex vortices in the vacancy dominated regime, we modified the $\mathrm{HC}$ model by imposing the additional constraint $a_{i} b_{i}=0$ on (6) and introducing the chemical potentials term $-\mu_{1} N_{A}-\mu_{2} N_{B}$ for each component to control the filling factors. Here one can expect [5] a long range phase separation as well as a short scale fluctuative phase separation corresponding to minority particles acquiring large cloud of vacancies.

If $1-N_{B}>N_{A}$, it is convenient to introduce a description in which the vacuum corresponds to all sites filled by $B$ particles. Then, the number $n=1-N_{B}$ of $B$ holes is shared between $N_{A}$ atoms and remaining $x_{v}=n-N_{A}>$ 0 vacancies. In the limit $N_{A} \ll N_{B} \sim 1$ transport of vacancies can be considered as transport of $B$ holes with the effective Hamiltonian

$$
H=\sum_{\langle i j\rangle}\left[-t_{1} a_{i}^{\dagger} a_{j} v_{j}^{\dagger} v_{i}-t_{2} v_{j}^{\dagger} v_{i}+\text { H.c. }\right],
$$

where $v_{i}^{\dagger}, v_{i}$ are the Pauli operators for the $B$ holes. In the mean field approximation, one should replace the field operators $a, v$ by the functions $a=\sqrt{x_{1}} \exp \left(i \varphi_{1}\right), v=$ $\sqrt{x_{v}} \exp \left(-i \varphi_{2}\right)$ with the slowly varying phases and perform the gradient expansion. (The minus in front of $i \varphi_{2}$ 


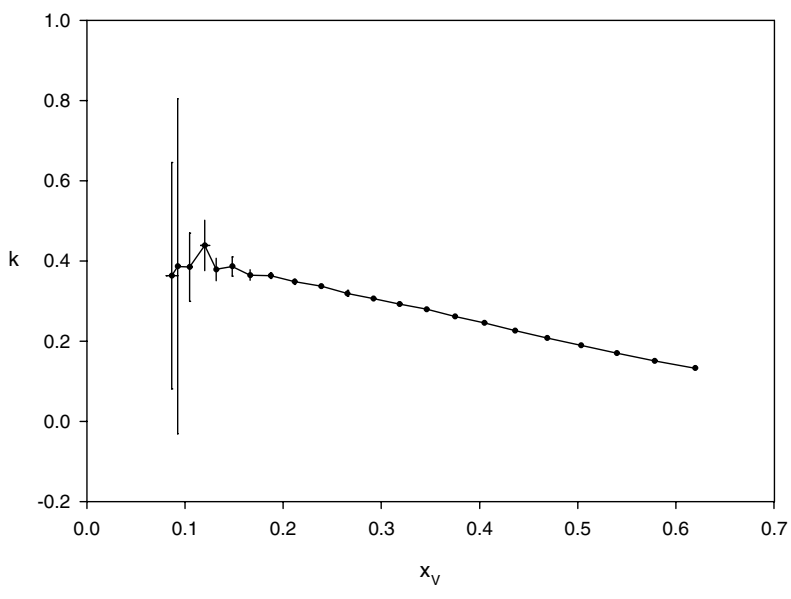

FIG. 2. The drag coefficient $k$ for the $J$-model analog of the Hamiltonian (6) in the limit $V_{\text {int }} \rightarrow \infty$ as a function of concentration of vacancies $x_{v}$ for symmetric case: $N_{A}=N_{B}, t_{a}=t_{b}$. Error bars are shown for all points. Solid line is the eye guide.

indicates that flow of holes and actual flow of mass are opposite.) This automatically generates the term $\sim t_{1} x_{1} x_{v}\left[\nabla\left(\varphi_{1}+\varphi_{2}\right)\right]^{2}$ in the effective energy from the first term in Hamiltonian (7). Obviously, the ratio of the stiffnesses becomes $k=\rho_{12} / \rho_{11}=1$ which corresponds to positive cross term typical for the vacancy-assisted transport meaning that the mean field captures well the physics of the vacancy-assisted transport. However, the prediction $k=1$ and $q=1$, is not supported numerically.

The Worm algorithm [14] Monte Carlo simulations in the HC limit with partial filling are similar to those described above (6) with an additional requirement of no double occupancy. We have found (Fig. 2) that, for $x_{v} \gtrsim$ $0.15, k<0.42 \pm 0.02$, and, thus, no topological complexes can exist as the lowest energy topological excitations in this regime. We do not have a simple explanation for this variance between numerical and mean field results. Most likely, the mean field result is not applicable for large $x_{v}$ in the symmetric mixture when the vacancies cannot be uniquely identified with the holes in the majority component.

As the number of vacancies is tuned to become $x_{v} \leq$ 0.15 , all stiffnesses exhibit large error bars which can be attributed to the regime of strong quantum fluctuations [5] associated with the degeneracy of the ground state in the $\mathrm{HC}$ limit. The precise nature of this effect requires separate analysis. For finite $V_{\text {int }}$, depending on $N_{A}, N_{B}$, the ground state can exhibit various types of ordering including the checkerboard insulator [8]. Then, decreasing $x_{v}$ at $N_{A}=$ $N_{B} \rightarrow 0.5$ will result in the first order phase transition with strong fluctuations, similar to those in Fig. 2, due to the domain formation.

Fractional q. - In the case of finite drag with $|k|<0.5$ fractional phase circulation $q=k$ can be observed when persistent current is interrupted by a Josephson junction which lifts the requirement of the integer of $2 \pi$ windings by creating a phase jump across the junction. Then, the phase winding is determined solely by the minimization of energy.

Detection. - The $(q+1)$ vortex complexes can be observed by absorptive imaging technique similar to imaging of vortices in one-component Bose-Einstein condensates [17]. A typical pattern should include extra $q$ fringes in one component.

In summary, we explored generic mechanisms of the drag effect in quantum bosonic mixtures in optical lattice with hard- and soft-core interaction. Strong mutual drag can result in composite topological structures. The drag in lattice is not controlled by particle effective masses. The simplest mean field approximation does not adequately describe the strong drag.

The authors acknowledge useful discussions with E. J. Mueller, N. V. Prokof'ev, and B. V. Svistunov as well as the support by the Grants No. NSF PHY-0426814, No. PSCCUNY (A. K.), and No. NSF DMR-0077266 (A. M.).

[1] M. Greiner et al., Nature (London) 415, 39 (2002).

[2] M. P. A. Fisher et al., Phys. Rev. B 40, 546 (1989).

[3] E. Demler and F. Zhou, Phys. Rev. Lett. 88, 163001 (2002).

[4] A. B. Kuklov and B. V. Svistunov, Phys. Rev. Lett. 90, 100401 (2003).

[5] A.E. Meyerovich, Phys. Rev. A 68, 051602(R) (2003).

[6] A. Kuklov, N. Prokof'ev, and B. Svistunov, Phys. Rev. Lett. 92, 030403 (2004).

[7] A. Kuklov, N. Prokof'ev, and B. Svistunov, Phys. Rev. Lett. 92, 050402 (2004).

[8] L.-M. Duan, E. Demler, and M. D. Lukin, Phys. Rev. Lett. 91, 090402 (2003).

[9] A. F. Andreev and E. P. Bashkin, Sov. Phys. JETP 42, 164 (1976) [Zh. Eksp. Teor. Fiz. 69, 319 (1975)].

[10] A. E. Meyerovich, Sov. Phys. JETP 60, 741 (1984) [Zh. Eksp. Teor. Fiz. 87, 1293 (1984)]; Progress in Low Temperature Physics, edited by D.F. Brewer (NorthHolland, Amsterdam, 1987), Vol. 11, pp. 1-73; SpinPolarized Phases of ${ }^{3} \mathrm{He}$, in: Helium Three, edited by W. P. Halperin and L. P. Pitaevski (Elsevier, New York, 1990), p. 757.

[11] D. V. Fil and S. I. Shevchenko, Low Temp. Phys. 30, 770 (2004).

[12] E. Timmermans et al., Phys. Rev. Lett. 83, 2691 (1999).

[13] E. Mueller (private communication).

[14] N. V. Prokof'ev, B. V. Svistunov, and I. S. Tupitsyn, Sov. Phys. JETP 87, 310 (1998); N. Prokof'ev and B. Svistunov, Phys. Rev. Lett. 87, 160601 (2001).

[15] M. Wallin et al., Phys. Rev. B 49, 12115 (1994).

[16] D. M. Ceperley and E. L. Pollock, Phys. Rev. B 39, 2084 (1989).

[17] E. L. Bolda and D. F. Walls, Phys. Rev. Lett. 81, 5477 (1998); S. Inouye et al., Phys. Rev. Lett. 87, 080402 (2001). 